\title{
USING MOBILE DEVICES FOR TOPOLOGICAL INFERENCE OF INDOOR ENVIRONMENTS
}

\author{
Marco Paiva ${ }^{1}$, Marcelo Petry ${ }^{1,2}$ and Rosaldo J. F. Rossetti ${ }^{1}$ \\ ${ }^{1}$ Artificial Intellegence and Computer Science Laboratory, Department of Informatics Engineering, \\ Faculty of Engineering, Univeristy of Porto, Rua Dr. Roberto Frias, S/N, 4200-465 Porto, Portugal \\ ${ }^{2}$ INESC Porto, Institute for Systems and Computer Engineering of Porto, \\ Faculty of Engineering, Univeristy of Porto, Rua Dr. Roberto Frias, S/N, 4200-465 Porto, Portugal \\ \{ee04127, marcelo.petry, rossetti\}@fe.up.pt
}

Keywords: Indoor Localization, Local Positioning Systems, Topology Mapping.

\begin{abstract}
Nowadays location systems are used within a large variety of applications. The application of these systems within indoor environments is already provided by several solutions. However, the need for high accuracy within these environments to pursue such purpose implies the use of specific infrastructures designed towards it. Our project tries to meet the requirements for a simple, low-cost, and scalable location system through different approaches. The main idea of it is to re-construct topological maps of indoor spaces through location estimation, i.e. using off-the-shelf technologies. We try to perform location estimations and then re-create the indoor maps as topological maps as a means of reducing the precision requirements other systems have, and develop a scalable and highly applicable system using sensors featuring mobile devices.
\end{abstract}

\section{INTRODUCTION}

Location-aware services have become an important area for today's ubiquitous and pervasive computing applications. The increasing use of such services brought the need to implement them in mobile platforms. Thus, location estimation systems that can provide those services in indoor environments have become subject of several studies and developments.

Today's solutions provide location-aware services in applications that are oriented towards localization in maps that give a metric perception of environments; thus, maps resemble their real environment. However, we believe that this metricoriented approach is not the optimum approach for a scalable implementation of indoor location-aware services used in mobile devices. In this sense, topological mapping approaches bring an abstraction to that metric information and are more likely to deal with low accurate measurements. In addition, they could be implemented with techniques that use less a priori information and in systems with limited computational power. This might be a good approach to improve scalability in such systems.

These two approaches are well studied in robotics and their development has been made based on the SLAM (simultaneous localization and mapping) methodology, for instance. While metric maps try to precisely represent the world by measuring and relating distances, as it is proposed in (Yiguang et al., 2010), the main idea behind the topological approach is to provide distinctiveness for global localization and mapping within the environment as it is done in (Beeson et al., 2005) or in (Sogo et al., 2001). More specifically, topological maps provide a sketch map with labelled places and abstract paths between them, in contrast to the metric maps that try to sketch all the features within the environment with geometric precision. A topological map approach can be used also in location-aware services oriented towards human utilization. For instance, humans would not require geometric locations to navigate in indoor environments and could easily do it through semantic locations and connections between them. The same idea can be applied to many intelligent systems which make use of location-aware services.

Our premise is to depart from a system with very little information about the environment. No map and topology is known a priori. Thus, its goal must be to achieve localization in the indoor environment and through that localization start building a topological map of the environment along the time and through a collaborative fashion among users. 
In order to materialize this idea, we have to emphasize the available sensor technologies that can be used to perform localization in indoor environments. The first technology that comes to mind when thinking about localization is GPS (Global Positioning Systems). However, this technology has a poor performance in indoor environments due to the lack of satellite coverage. Some works have been made also with cellular networks, namely using GSM (Global System for Mobile Communication), but it suffers from the same effects as referred to GPS. Nevertheless, better performances can be achieved through the use of $a$ priori data about the environment, situation which we intend to minimize in our research.

One of the most explored technologies to perform localization in indoor environments is WiFi Wireless LAN. This is a technology widely implemented in public infrastructures and that is also built-in in today's ordinary mobile devices. However, this kind of technology suffers from significant signal attenuation effects in indoor environments, such as: multipath; refractions; reflections; scattering and shadowing. These problems are normally overcome through fingerprinting approaches, as in GPS or in GSM. However, several techniques can be performed without fingerprinting methods and using Wi-Fi WLANs. These methods rely on simple lateration or angulation techniques or even doing an on-line calibration phase similar to fingerprinting.

Another technology that can be used for localization purposes and that is widely spread in mobile devices is Bluetooth. Despite similar to Wi$\mathrm{Fi}$ in some aspects, Bluetooth measurements are only able after establishing a connection between devices. This is usually overcome through learning algorithms, such as Artificial Neural Networks to estimate device positions. Another approach relies on using other sensor technologies that are also highly available on today's mobile devices, such as accelerometers, magnetometers and gyroscopes. Such an approach usually performs dead-reckoning of devices when the users are on the move through acceleration and orientation measures.

We are specifically interested in studying the potential of available technology to support easy and practical inference of topological maps for indoor applications. We want to provide some guidelines to select mobile-enabled sensors to base topological map inference methods, and build such maps through localization of devices in indoor environments in a collaborative fashion. With the localization estimates of many devices we expect to achieve a better basis for performing topological map inference methods.

Firstly, we analyse the best techniques and technologies to perform localization with mobile devices, which can provide us with a better basis for topological map inference through simple, low-cost and common infrastructures. Secondly, we show how such a system is implemented in those devices and make an analysis of the preliminary implementation and results using multiple devices.

\section{RELATED WORK}

Several works have been developed in order to achieve localization of mobile devices in indoor environments. Topological map inference has been made mainly in the robotics field, focusing on the SLAM methodology, as we mentioned before. Nevertheless, we must first focus on localization issues for mobile devices.

\subsection{Solutions for Localization Applications}

Several GPS-based systems and approaches have been proposed for localization purposes. SnapTrack (Biacs, 1999), uses A-GPS and achieves accuracies between $5-50 \mathrm{~m}$. However, GPS is not the ideal technology to use in such a purpose due to its poor performance in this kind of environments. With GSM based systems, (Otsason et al., 2005) presented a solution that led to an accuracy as low as $2.5 \mathrm{~m}$. The main idea of it was to use wide signalstrength-fingerprints in a training phase, what implies several a priori configurations within the environment. This is a down point regarding the idea of using as less a priori configurations as possible to perform localization with a specific system, as we intend to do.

Lots of Wi-Fi-based solutions have been also developed and we give emphasis to two of them. The RADAR solution proposed in (Bahl and Padmanabhan, 2000), which is one of the most cited systems in related literature, provides localization through two main ways. One work is in a basic fingerprinting approach where several off-line measurements are made in a prior training phase, whereas the other is based on a signal propagation model. The approaches use the $k$-nearest neighbour algorithm to achieve positioning estimations. The number of neighbours, the number of off-line measured points, user orientations, and the number of samples achieved in the real-time measurement 
phase affect the accuracy of the system. They are reported to achieve accuracy around 2-3m. Another work (Ekahau, 2010) is one of the most accurate systems that use the Wi-Fi sensor technology. It uses a Bayesian approach combined with stochastic complexity and a competitive online learning algorithm. Although it achieves accuracy as high as $1 \mathrm{~m}$, it is achieved through a training phase that implies a strict calibration that has to be performed in a very complex and precise way.

We also found Bluetooth solutions in our literature review. The one presented in (Tadlys, 2010) reported an accuracy of about $2 \mathrm{~m}$, with $95 \%$ reliability with a positioning delay between 1530sec. The solution relies on wireless tags and wireless access points. The use of inertial sensors as referred in the Introduction finds also some developed solutions in literature. They are mainly done through a pedestrian tracking approach using a proper algorithm to estimate the device's orientation and in a next phase they take samples of the accelerometer sensor to acquire the mobility of the device. In (Yiguang et al., 2010), estimation is made according to a walking pattern that provides a realistic representation of movements of a human carrying the device. Reported results have shown a better trend with an increase of devices being tracked and given errors between 2-3m on the floor map estimation, not considering angle errors.

\subsection{Localization Techniques}

The main techniques used on systems that perform localization estimation in indoor environments nowadays are identified in the next sub-section.

\subsubsection{Lateration and Angulation Methods}

Lateration is a technique that can provide a position estimate of a mobile device through at least three measures for the two dimensional case. Essentially it is the conjunction of three distances between transmitters and one receiver that can provide the position through lateration. Given those distances, it is possible to know that the receiver is within three circumferences, on which their radius are the distances computed between transmitters and receiver. The localization of the receiver can be then achieved through the Euclidean distance. \#he best case scenario of this technique is when the three circumferences intercept themselves at one point. In this case the estimated receiver position would be that point. However, it is possible that the circumferences do not intercept at a single point, being the estimation through this technique not precise or even impossible to define. This is basically due to error estimations on the distance between reference points and the device, which exists due to the use of propagation models that cannot optimally describe the environment characteristics. To overcome this problem leastsquares algorithm is usually applied to achieve a better estimation of the receiver position.

On the other hand, angulation takes advantage of the "angles" of the received signal on transmitters. Basically, angulation only requires to get two reference-point transmitters and measuring their AoA (angle of arrival). With those values it is possible to sketch a triangle and then obtain the position of the device (Hui et al., 2007).

\subsubsection{Scene Analysis}

Scene analysis makes use of a predefined data set that maps observed features into object locations. One example of static scene analysis is the RADAR system, presented in (Bahl and Padmanabhan, 2000). This technique is considered one of the best alternatives to simple triangulation or lateration methods made without a priori fingerprinting. In theory, the static scene analysis can be performed with all the measurements or features available. But for indoor localization purposes, the most common metric used is RSSI (received signal strength indicator). Other metrics can also be achieved and used, for example AoA (angle of arrival) or ToA (time of Arrival), or even the use of image analysis for navigation, which can be done also through mobile platforms with built-in cameras.

Scene analysis is usually performed in two phases: one training phase that is usually made offline, and where normally a database is built containing pattern variations of one or more features in known locations; and, another phase when the location estimation is done through a relationship analysis with data originated in the first phase. Deterministic and Probabilistic models are normally used to perform the matching in the second phase.

The main advantages of scene analysis concern the fact that location of "objects" can be inferred using passive observations. However, this kind of method requires previous analysis of scenes, so that a comparison can be performed to achieve location.

\subsubsection{Using Neural Networks}

Artificial Neural Networks (ANN) is another approach to yield indoor localization, as it is done in (Ahmad et al., 2006). The basic concept is to use 
ANN to produce a non-linear input-output mapping. It is used in several applications, such as classification, approximation or regularization. This kind of approach is similar to scene analysis but, instead of using probabilistic methods, it uses ANN to compute the matching between the fingerprints and the real time observations. The key advantage of using ANN is that it has a lower cost in terms of time and space necessary to process measurement acquisitions. However, as this kind of approach mostly relies on learning algorithms, it can induce to some erroneous estimation. ANN is also well suited for different complex and dynamic indoor environment estimations.

\section{A PRELIMINARY ASSESSMENT}

To implement a system that could be applied on scalable basis, which would not require as much $a$ priori setups, one that could be provided through ordinary mobile devices and within common infrastructures, we opted to develop a solution using three main sensor technologies: Wi-Fi sensors within WLAN, performing a lateration technique with a minimum squares optimization procedure, accelerometers and magnetometers to perform dead reckoning with the mobile devices through a pedometer and a compass emulation.

The Wi-Fi sensors and the performed lateration technique was chosen due to the high availability that this kind of sensors have in existing infrastructures and also due to the low complexity characteristic of the lateration technique that does not require much setup data to be performed. Nevertheless, we need to remark the low accuracy in localization estimation that this technique has. However, our goal was not to achieve the most accurate estimation but to be able to construct topological maps which do not require precise metric information about the environment.

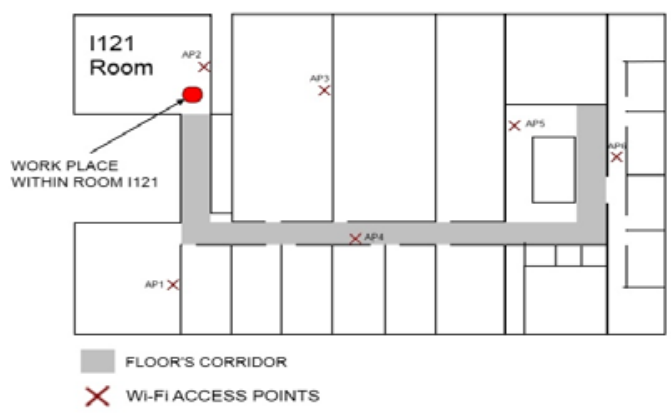

Figure 1: Floor plan where the experiments were made.
We chose the accelerometers and magnetometers to perform dead-reckoning also due to their high availability in today's mobile devices. To perform the dead-reckoning we make use of the pedometer emulator to count steps, and compute the orientation of each step through the fusion of an accelerometer and a magnetometer from where we are able to emulate a compass.

In order to implement the system we developed an application under the Android OS to be used on mobile phones, and which had the task to collect data from all those sensors. Then we performed the localization estimations outside the application and through that collected data. We must remark that we perform estimations outside the mobile devices because our idea is to provide a collaborative solution among users in order to accomplish a more reliable map reconstruction.

\subsection{Wi-Fi Lateration Results}

\subsubsection{Path Tracking Estimations of Moving Devices}

In this experiment we performed several estimations of five different devices within a corridor path in the floor presented in Figure 1. The results of all merged estimations achieved accuracies as low as $1.4 \mathrm{~m}$ to the real corridor path. However, these results presented a maximum deviation of $7.37 \mathrm{~m}$ in some of the estimations.

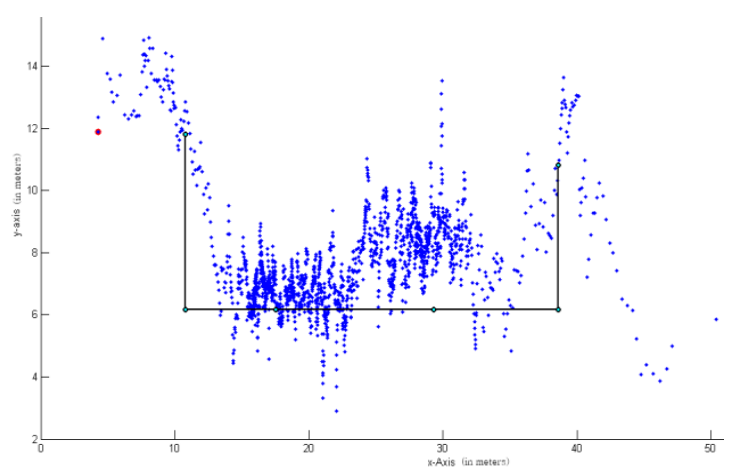

Figure 2: Localization estimations made along a walking path with Wi-Fi, after applying a Kalman filter.

Figure 2 presents merged estimations along that path; blue dots are estimations achieved after a Kalman Filter procedure applied over raw estimations, and the line in black represents the real path, where the mobile devices moved. 


\subsubsection{Dead-Reckoning Estimation Made within a Path}

In this experiment we carried out dead-reckoning within the same path illustrated in Figure 1. As the orientation estimations made had too much magnetic influence from the environment, we filtered the measurements through a median filter and clustered the orientations to a minimum orientation change of $90^{\circ}$, as all the corridors within the environment can be modelled like that.

The results presented an improvement of the accuracy in comparison to the estimations made with the Wi-Fi lateration, as low as $1.05 \mathrm{~m}$ with a maximum error deviation of 3.0m.

In Figure 3, we illustrate one of the results achieved with one mobile device where the line in black is the walking path and the blue dots are the estimations made with dead-reckoning.

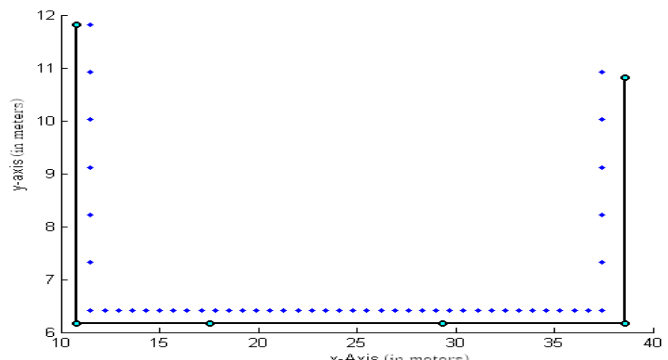

Figure 3: Estimation of the walking path through deadreckoning.

\subsubsection{Inference of a Corridor Topology and a Room Space}

This was the inference experiment that we made using the previously described results. Its main goal was to infer a single room as a starting point and the corridor that connects to that room. To do it we used a histogram of the estimations made with the $\mathrm{Wi}-\mathrm{Fi}$ sensor and which were gathered in the I121 room, which is illustrated in Figure 4.

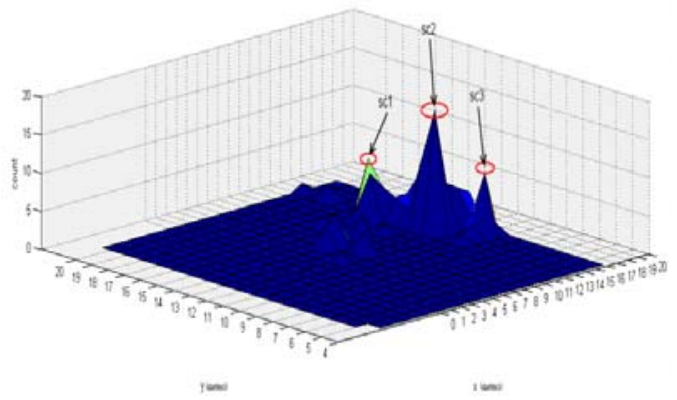

Figure 4: Wi-Fi lateration estimations histogram, performed in room I121.
With that histogram we computed a single position which corresponds to the centre of mass between the 3 positions with more estimations along the time (sc1, sc2, sc3) as it is depicted in Figure 4. Thus, we inferred it as the working place within the room; we used the dead reckoning estimations, starting at that single position and performed along the corridor topology.

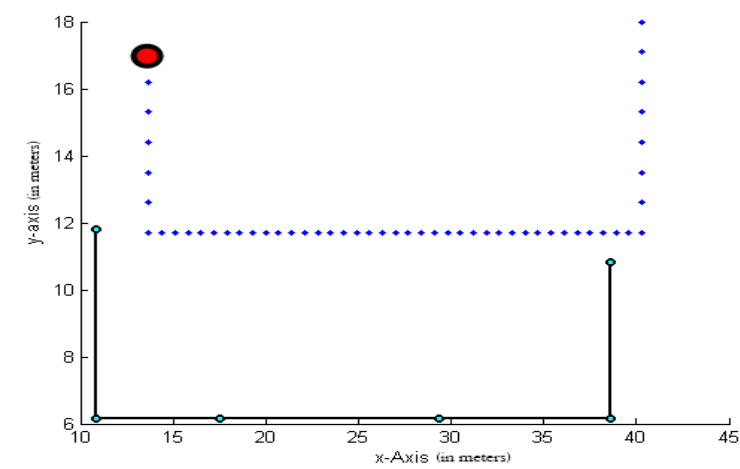

Figure 5: Inference of a room connected to the corridor topology.

With those estimations we inferred the corridor topology and gathered the working place within room I121. When fusing the two inferences, we were able to achieve the inference of the room connected to the corridor as it is shown in Figure 5, where blue dots are the dead-reckoning estimations and the red circle is the working place estimated with the histogram. However, the sketched topology is deviated from the real one due to the error of the estimation made with the $\mathrm{Wi}-\mathrm{Fi}$ at the position in the room. Despite this deviation, the topology is similar to the real one and we can conclude that it is a good inference made within the topology map purpose we pursue in our research.

This method can be applied to several semantic locations and extended into different environments, as they can be inferred based on the time users spend in different spots.

\section{CONCLUSIONS}

In order to accomplish the goals of this work we developed a system using sensor technologies featured on the most common mobile devices: Wi-Fi sensors, accelerometers and magnetometers. With the application we developed we were able to gather important data to perform localization within indoor environments. We should first remark the low accuracy that we achieved with the Wi-Fi lateration 
estimations, which at a first glance can lead us to thinking in the unfeasibility of such a purpose using this technique. However, when looking into the overall results gathered with the Wi-Fi lateration and the dead-reckoning made with the accelerometer and the magnetometer, the achieved results led us to conclude the feasibility of creating an indoor environment topological map with those localization estimations over the time they are gathered. In this sense we were able to make a simple inference where we achieved a topological map of a room connected to a corridor which was very realistic and which reinforces it is possible to accomplish our purpose.

As next steps or further developments that can be taken after this work, we might suggest the improvement that can be made in order to achieve even more localization estimations, which can be made for instance by adding more sensor technologies to the system, such as Bluetooth. Another kind of improvement can be made in the scope of optimizing the inference methods. This can be achieved, for example, by using regression models or clustering techniques to estimate the topology of the indoor environment using all the estimations gathered with all the sensors at the same time.

As future applications of our work we should state that it leads to the suggestion of several future studies within the area of local positioning systems. Navigation applications, such as the ones used nowadays within outdoor environments but applied to indoor spaces are one of the ways of exploring the developments of this project. Integration with autonomous driving systems or other applications in the robotics field of research is another possibility. Integration with applications used for indoor commercial purposes, as a way to provide better efficiency and easier functionalities when consumers search for products within indoor areas is also considered. Using such a system in rescue situations, as a way to know in advance the topology of the rescue environment represents a very promising field of application as well. Location-based social games are another area of great potential for this work. Generally all applications that make use of indoor topology maps can benefit from a system that provides, through inference, indoor topology maps.

\section{REFERENCES}

Ahmad, U., Gavrilov, A., Nasir, U., Iqbal, M., Seong Jin,

C. \& Sungyoung, L. (2006). In-building Localization using Neural Networks. In: IEEE International Conference on Engineering of Intelligent Systems, 2006. p.1-6.

Bahl, P. \& Padmanabhan, V. N. (2000). RADAR: an inbuilding RF-based user location and tracking system. In: Proceedings of the 19th Annual Joint Conference of the IEEE Computer and Communications Societies (INFOCOM). vol.2, p.775-784.

Beeson, P., Jong, N. K. \& Kuipers, B. (2005). Towards Autonomous Topological Place Detection Using the Extended Voronoi Graph. In: Proceedings of the 2005 IEEE International Conference on Robotics and Automation (ICRA). p.4373-4379.

Ekahau (2010). Ekahau, Wi-Fi Based Real-time Tracking and Site Survey Solutions? [Online]. Available: www. ekahau.com [Accessed on 26/Feb./2011].

Hui, L., Darabi, H., Banerjee, P. \& Jing, L. (2007). Survey of Wireless Indoor Positioning Techniques and Systems. IEEE Transactions on Systems, Man, and Cybernetics, Part C: Applications and Reviews. (37): 1067-1080.

Otsason, V., Varshavsky, A., Lamarca, A. \& De Lara, E. (2005). Accurate GSM Indoor Localization. In: Beigl, M., Intille, S., Rekimoto, J. \& Tokuda, H. (eds.) UbiComp 2005: Ubiquitous Computing. Springer.

Sogo, T., Ishiguro, H. \& Ishida, T. (2001). Acquisition and propagation of spatial constraints based on qualitative information. IEEE Transactions on Pattern Analysis and Machine Intelligence. (23):268-278.

Tadlys (2010). Topaz local positioning solution [Online]. Available: www.tadlys.com [Accessed on 25/Feb./ 2011].

Yiguang, X., Sengupta, R. \& Fallah, Y. (2010). Making indoor maps with portable accelerometer and magnetometer. In: Ubiquitous Positioning Indoor Navigation and Location Based Service (UPINLBS). p.1-7.

Biacs, Z., Marshall, G., Moeglein, M., Riley. W. (1999). The Qualcomm/ SnapTrack Wireless-Assisted GPS Hybrid Positioning System and Results from Initial Commercial Deployments. In: Proceedings of the 55th ION Annual Meeting, Navigational Technology for the 21st Century. 